\title{
La polarización de la política de salud en México
}

\author{
Health policy polarisation in Mexico
}

Oliva López-Arellano 1

José Blanco-Gil 1

1 Maestría en Medicina Social, Universidad Autónoma Metropolitana-Xochimilco. Calzada del Hueso 1100, Col. Villa Quietud, Coyoacán, CP 04960, México DF, México. oli@cueyatl.uam.mx jblanco@cueyatl.uam.mx

\begin{abstract}
In the last 17 years, health policy in Mexico has been shifted from a conception of integrated health care and a gradually extended coverage as a major responsability of the State and health care public institutions, to in the one hand, a very active promotion of market and private profit in health services and in the other, poverty relief programs. In this paper we identify different periods corresponding to the last three presidential terms. Each clearly represent different stages of health sector reform: transitional (1982-1988), mercantilisation and poverty relief (1988-1994) and, strengtheing of the so called health markets (1994-2000). The analised transformation is part of the set of secundary reforms subordinated to the structural adjusment and the economic and social megaprojects impossed by the international financial intitutions.

Key words Health Policy; Privatisation; Health Polarisation
\end{abstract}

Resumen En los últimos 17 años, la política de salud en México ha transitado de una concepción de atención integral y de extensión gradual de coberturas como responsabilidad del Estado, a través de las instituciones públicas de salud, a una activa promoción gubernamental de la mercantilización de servicios, complementada con una política de beneficencia hacia la población pobre. En esta transformación se identifican tres periodos que se corresponden con los sexenios presidenciales y que expresan tres momentos distintos de la reforma del sector salud: el primero (1982-1988) caracterizado como transicional, el segundo (1988-1994), en donde emerge con claridad la estrategia bipolar de mercantilización y beneficencia y el tercero (1994-2000), en donde las estrategias gubernamentales se orientan a fortalecer los mercados de la salud. Esta reestructuración del sector salud ha sido instrumentada en forma explícita desde 1982 y forma parte del conjunto de reformas secundarias derivadas de la adecuación subordinada del campo social a las políticas de ajuste estructural y a los megaproyectos económicos y sociales impuestos por los organismos financieros internacionales.

Palabras clave Política de Salud; Mercantilización; Polarización en Salud 


\section{Introducción}

En México, a partir de 1982, las políticas de ajuste estructural adoptadas para remontar la crisis económica tienen un severo impacto sobre el financiamiento y la organización de las instituciones de bienestar social en general y de salud en particular, frenan la tendencia expansiva de los servicios públicos de salud, limitan su lógica redistributiva, debilitan su capacidad de respuesta y posibilitan la modificación de sus tendencias universalistas, solidarias e integrales, claramente incompatibles con las propuestas de modernización neoliberal de la política social (Soria \& Farfán, 1990; López \& Blanco, 1993).

En sentido inverso a la complejidad de las condiciones riesgosas para la salud (Tablas 1, 2 y 3) y al incremento de las desigualdades de enfermedad y muerte entre distintos espaciospoblación (Tabla 4), las políticas de salud se redefinen y simplifican a partir de los ejes de la modernización neoliberal, justificando un conjunto de reformas de los servicios de atención médico-sanitaria que, lejos de responder a la diversidad epidemiológica (Tabla 5), fragmentan y limitan el quehacer público en salud (Blanco et al., 1998). Esta reformulación del campo sanitario se expresa en las prioridades explicitadas en el discurso gubernamental, en las estrategias seleccionadas para reorganizar el sector, así como en el monto y destino de los recursos financieros.

La política de salud se debate en la contradicción que permea a todo el quehacer estatal en el mundo globalizado: por una parte, apoyar activamente los procesos de expansión del capital, privatizando lo público rentable, y, por otra regular el conflicto social a través de las instituciones ejecutoras de las políticas sociales. Esta contradicción conduce a refuncionalizar la acción pública de modo que se corresponda con el proyecto de reestructuración económica global y, al mismo tiempo, se inscriba en la búsqueda de consensos. Se delinea así una propuesta eficientista de la política social que racionaliza los recursos, intenta abaratar los costos, simplifica al máximo la prestación de los servicios y redefine las poblaciones a las que se orienta. En forma progresiva, se fomenta - desde el gobierno - la participación de la iniciativa privada en la producción de servicios que suponen una mayor rentabilidad económica, se plantea su regulación a través de las fuerzas oligopólicas del mercado y, paralelamente, se profundiza la acción selectiva del Estado a través de programas de administración de la pobreza, reeditando una versión moderna de la beneficencia decimonónica (López \& Blanco, 1993).

\section{El periodo 1982-1988}

El sexenio del presidente Miguel de la Madrid (1982-1988) constituye un período de transición que sienta las bases para la llamada Reforma del Estado. La política de salud - como ámbito particular de la política social - también se redefine, como resultado de la contradicción existente entre el aumento de costos para la atención médica, el decremento del presupuesto asignado al sector y la necesidad del Estado mexicano de "modernizar" su intervención en el ámbito de la regulación, del financiamiento y de la producción de servicios de salud.

En 1983, se eligen las estrategias de: modernización administrativa, sectorización, coordinación intersectorial, descentralización y participación comunitaria, con el propósito de conformar un Sistema Nacional de Salud (SNS) que permita incrementar la eficiencia de los servicios y racionalizar los recursos. El objetivo general del SNS es tender hacia una cobertura nacional de los servicios de salud, garantizando un mínimo razonable de calidad y una mejoría en el nivel de salud de la población (Soberón \& Kumate, 1988). Para avanzar en este objetivo, se realiza una intensa actividad legislativa, cuyo punto de partida es la modificación del Artículo 4o que eleva a rango constitucional el derecho a la protección a la salud y la aprobación de la Ley General de Salud que entra en vigor en julio de 1984 (Secretaría de Salud, 1984). Es importante destacar que el Derecho a la Salud ya existía en la Constitución Mexicana y que la reforma al Artículo 4o consiste en señalar la protección a la salud como derecho individual y como responsabilidad del Estado.

A pesar de ser una propuesta viable para reordenar el sector, el SNS se debate entre los límites impuestos por el proyecto neoliberal (disminución del gasto social, apertura de nuevos espacios para la acumulación de capital, privatización selectiva de lo público rentable, desregulación) y los procesos de legitimación y búsqueda de consensos (derecho ciudadano a la protección de la salud, atención a grupos más desprotegidos, universalización de la cobertura de los servicios, aumento de la calidad de la atención, etc.).

En este contexto, los planteamientos iniciales sobre la protección a la salud como derecho de todos los mexicanos se desvirtúan al subordinarse la política social al proyecto de reestructuración económica y al prevalecer la lógica neoliberal en la conformación del SNS. Esta subordinación se expresa con claridad en el financiamiento y en la descentralización de los servicios. 
Tabla 1

Evolución de la pobreza. México, 1984-1999.

\begin{tabular}{|c|c|c|c|c|c|c|c|}
\hline \multirow[t]{2}{*}{ Año } & \multirow{2}{*}{$\begin{array}{c}\text { Población total } \\
\text { Habitantes } 1\end{array}$} & \multicolumn{2}{|c|}{ Pobreza extrema } & \multicolumn{2}{|c|}{ Pobreza moderada } & \multicolumn{2}{|c|}{ Población no pobre } \\
\hline & & Habitantes 1 & $\%$ & Habitantes 1 & $\%$ & Habitantes 1 & $\%$ \\
\hline 1984 & 71,4 & 10,71 & 15 & 19,3 & 27 & 41,4 & 58 \\
\hline 1992 & 84,3 & 13,48 & 16 & 23,6 & 28 & 47,2 & 56 \\
\hline 1999 & 94,5 & 26,50 & 28 & 14,2 & 15 & 53,8 & 57 \\
\hline
\end{tabular}

1 En millones.

Fuente: Grupo Financiero Banamex-Accival, 2000.

La descentralización de los servicios de salud se constituye - en el periodo 1984/88 - en la estrategia de mayor importancia para reorganizar el subsistema de atención a población no derechohabiente. La postura gubernamental señala que a través del proceso descentralizador se pretende ampliar las coberturas, mejorar la calidad de los servicios y conciliar la eficiencia en la prestación de la atención y los derechos de los trabajadores. Asimismo, se enarbola un discurso federalista, que plantea revertir el proceso centralizador y trasladar la responsabilidad de la operación, la capacidad de decisión y los recursos, al lugar donde se efectúa la prestación de los servicios en el marco de los sistemas estatales de salud. Otros propósitos que rápidamente adquieren gran importancia son: el abaratamiento de costos, la racionalización del gasto en salud y la suma de esfuerzos financieros.

En este proceso, se pretende transferir responsabilidades a la periferia, comprometiendo a los niveles regionales y locales en la resolución financiera, organizacional y técnica de los problemas. Se intenta, también, desarticular estructuras sindicales de alcance nacional que se constituyen en obstáculos para la flexibilización de las relaciones laborales de los trabajadores de la salud. En esta dinámica se manifiestan los nuevos derroteros de la concertación social, en donde el "pacto" social Estado-clase obrera pierde importancia (Barba, 1997). El proceso tiene dos vertientes, la desconcentración de las facultades a los Servicios Coordinados de Salud Pública en los Estados (SCSPE) y en el Distrito Federal (SCSPDF) y la descentralización propiamente dicha, a través de la creación de Sistemas Estatales de Salud y de la transformación de la Secretaría de Salud en una dependencia normativa, de supervisión, programación y evaluación (Espinoza, 1986).

La coordinación programática - primera fase de la estrategia descentralizadora - se em-
Tabla 2

Distribución del ingreso por deciles de hogares. México, 1984-1998.

\begin{tabular}{lccccc}
\hline Deciles de hogares & 1984 & 1989 & 1994 & 1996 & 1998 \\
\hline I-VI (Pobres) & 28,6 & 26,1 & 25,4 & 26,9 & 25,5 \\
VII-IX & 38,6 & 36,0 & 36,2 & 36,5 & 36,4 \\
X & 32,8 & 37,9 & 38,4 & 36,6 & 38,1 \\
Coeficiente de Gini & 0,429 & 0,469 & 0,477 & 0,456 & 0,476 \\
\hline
\end{tabular}

Fuente: Boltvinik, 2000a.

prende en 31 estados y alcanza diversos grados de avance. La integración orgánica se inicia en mayo de $1985 \mathrm{y}$, al finalizar ese año, el proceso abarca nueve entidades federativas (Nuevo León, Guerrero, Baja California Sur, Morelos, Tabasco, Jalisco, Querétaro y Sonora). En marzo de 1986, se suman otros tres estados (Colima, Guanajuato y México) y a fines de 1987, dos entidades más se descentralizan (Aguascalientes y Quintana Roo). En estos estados desaparecen los Servicios Coordinados de Salud Pública y el Programa de Solidaridad Social por Cooperación Comunitaria (IMSS-COPLAMAR) y se crean dependencias estatales responsables de los servicios. Sólo 14 estados finalizan la etapa de integración orgánica y descentralización operativa, por lo que, al término del sexenio, la descentralización de los servicios de salud queda inconclusa.

El avance de la descentralización en los estados es desigual y, en mayor o menor medida, enfrenta problemas de diverso orden. Entre los más importantes, se pueden mencionar: insuficiente asignación y disponibilidad de recursos financieros; limitaciones de infraestructura y de canales adecuados de distribución que generan desabasto de insumos y de equipo médico en el nivel jurisdiccional y saturan la capacidad de almacenamiento, conservación y man- 
Tabla 3

Evolución de los salarios mínimos y medios. México, 1982-1999. (Pesos de 1994 por día).

\begin{tabular}{|c|c|c|c|c|c|c|c|c|}
\hline \multirow[t]{2}{*}{ Tipo de salario } & \multicolumn{4}{|c|}{ Años } & \multicolumn{3}{|c|}{$\%$ de cambio sexenal } & \multirow{2}{*}{$\begin{array}{c}\% \text { de cambio } \\
\text { en el periodo } \\
1982-1999\end{array}$} \\
\hline & 1982 & 1988 & 1994 & 1999 & $1982-88$ & $1988-94$ & 1994-99 & \\
\hline Salario mínimo & 41,0 & 17,6 & 13,4 & 10,5 & $-57,1$ & $-23,9$ & $-21,6$ & $-74,4$ \\
\hline Industria textil & 52,5 & 31,0 & 27,5 & 21,2 & $-41,0$ & $-11,3$ & $-22,9$ & $-59,6$ \\
\hline Industria manufacturera & 137,0 & 73,0 & 102,4 & 79,7 & $-46,7$ & 40,3 & $-22,2$ & $-59,6$ \\
\hline Industria maquiladora & 69,7 & 48,8 & 55,0 & 51,5 & $-30,0$ & $-12,7$ & $-6,4$ & $-26,1$ \\
\hline Construcción formal & 55,5 & 33,2 & 42,6 & 29,9 & $-40,2$ & $-28,3$ & $-29,8$ & $-46,1$ \\
\hline Comercio menudeo & ND & 40,6 & 49,4 & 38,6 & ND & $-21,7$ & $-21,9$ & ND \\
\hline
\end{tabular}

$\mathrm{ND}=$ No disponible

Fuente: Boltvinik, 2000b

tenimiento de los niveles estatal y local; problemas laborales por la oposición de la Federación de Sindicatos de Trabajadores al Servicio del Estado (FSTSE); restricciones severas en la apertura de plazas y contratación de personal e imposibilidad para garantizar la homologación salarial y funcional, así como la negativa de los gobiernos locales a estatizar al personal de los servicios por las repercusiones económicas y políticas para los estados (López, 1990).

En el caso del Programa IMSS-COPLAMAR (actualmente IMSS-Solidaridad), los problemas se profundizan, pues la entrega de las unidades médicas a los SESA se realiza sin el soporte material, humano y logístico-administrativo proporcionado - hasta antes de la descentralización - por el régimen ordinario del Instituto Mexicano del Seguro Social (IMSS). El paro casi total de las actividades del Programa IMSS-Coplamar en los estados descentralizados y la redefinición de atribuciones dentro del sector salud confrontan a las cúpulas burocráticas del IMSS y de la Secretaría de Salud y explican por qué, al finalizar el proceso de descentralización en los 14 estados, el Programa IMSS-COPLAMAR sólo ha cedido el $28 \%$ de sus unidades, conservando una población potencial de 6,5 millones de habitantes (González \& Leyva, 1988; López, 1990).

La situación de desmantelamiento de los servicios es tan grave que, en 1988, se suspende indefinidamente el proceso descentralizador. El Programa IMSS-Solidaridad se recupera parcialmente y, para 1990, opera en 17 estados, con 2.666 unidades de primer nivel (UMR), 52 hospitales rurales y una cobertura aproximada de 11 millones de mexicanos (IMSS-Solidaridad, 1990a). Sin embargo, las condiciones para transitar hacia la fragmentación institucional y la focalización de las intervenciones sanitarias ya están dadas, y el discurso gubernamental las justifica en términos del ahorro y de la optimización del gasto social (Programa Nacional de Salud, 1990).

En el marco del "nuevo federalismo", se trasladan responsabilidades de la Federación a los estados sin fortalecer las capacidades financieras, administrativas, técnicas, de gestión de los niveles locales, sentando las bases para una redefinición político-económica que limita y fragmenta el quehacer público en salud y que permite instrumentar nuevos esquemas de financiamiento y producción de servicios con una lógica mercantil (Laurell, 1992).

La oposición de algunos gobiernos estatales, la insuficiencia de recursos financieros (federales y locales) para apoyar el proceso descentralizador y las pugnas de las cúpulas burocráticas institucionales determinan que el proceso descentralizador del periodo 1982-88 quede inconcluso. En los 14 estados en donde se concreta, se trata más de una desconcentración operativa que privilegia la atención de bajo costo y profundiza la inequidad en el acceso a servicios de salud de calidad (López \& Blanco, 1993).

La "horizontalización" de los programas verticales de control de enfermedades se plantea como parte de la reorganización del sector, con el propósito de ampliar las coberturas, aprovechar al máximo los recursos y obtener mayor rendimiento desde el punto de vista costo-beneficio, en un contexto de limitaciones financieras. Los programas son entregados a los servicios estatales para su operación, independientemente si están descentralizados o no.

Este proceso enfrenta diversos problemas, entre ellos: falta de capacidad técnica y financiera de los servicios estatales para asumir de manera integrada las acciones de cada programa; rigidez en los procedimientos administrativos que impide la gestión oportuna de recur- 
sos, equipo e insumos; resistencia de los trabajadores para incorporarse como personal "regular" bajo las jerarquías jurisdiccional y estatal; pérdida de movilidad y atomización de los equipos especializados por la adscripción de brigadas móviles a las unidades de salud; concentración del personal en las oficinas estatales y desatención de los niveles jurisdiccionales y/o locales, e insuficiencia de recursos financieros para garantizar la movilidad del personal operativo y supervisor, del material y del equipo (Blanco \& López, 1990).

Estos problemas se presentan en todos los estados, aunque la integración del personal y la continuidad de las actividades son variables, dependiendo fundamentalmente del nivel de desarrollo de la red de unidades y de la capacidad económica de los gobiernos estatales (Fernández-de-Castro, 1988). En los hechos, se produce un desmantelamiento temporal de los programas de control de enfermedades, con la consecuente reducción de la eficacia de las medidas epidemiológicas puntuales. La regresión sanitaria en este periodo puede ser ejemplificada con el incremento en la incidencia de paludismo, dengue y tuberculosis, con la epidemia de sarampión 1989-90; asi como con el incremento de la mortalidad por desnutrición en preescolares y con la profundización de las diferenciales de salud entre grupos y regiones (Blanco \& López, 1990).

\section{El periodo 1988-1994}

La política de Salinas de Gortari (1988-1994) asume plenamente las tesis neoliberales de la centralidad del mercado como organizador de la vida social y económica, en un contexto de profunda crisis política. El gobierno salinista avanza velozmente en el proyecto modernizador neoliberal y enfrenta la crisis política mediante un discurso "solidario" que progresivamente será definido como liberalismo social.

El Programa Nacional de Solidaridad (Pronasol) es la versión más acabada de la política social asistencialista y centralizada que caracteriza al sexenio (López, 1992). En este programa se propicia una relación casi directa entre la figura presidencial y la población pobre, al margen de las instituciones de bienestar social, debilitando a las instituciones públicas y violentando incluso las propuestas descentralizadoras del sexenio anterior que buscaban sentar nuevas bases geopolíticas para el desarrollo de la política social (Poder Ejecutivo Federal, 1988). Se profundizan el manejo discrecional de los recursos y la focalización de las acciones liga-

\begin{tabular}{|c|c|c|c|}
\hline \multirow[t]{2}{*}{ Causa de muerte } & \multicolumn{3}{|c|}{ Tasas de mortalidad por causa específica 1} \\
\hline & Nacional & $\begin{array}{l}\text { Estados com muy } \\
\text { alta marginación2 }\end{array}$ & $\begin{array}{l}\text { Estados com muy } \\
\text { baja marginación } 3\end{array}$ \\
\hline $\begin{array}{l}\text { Enfermedades } \\
\text { del corazón }\end{array}$ & 69,4 & 52,0 & 88,5 \\
\hline Tumores malignos & 52,6 & 43,8 & 62,9 \\
\hline Diabetes mellitus & 36,4 & 24,8 & 46,4 \\
\hline Accidentes & 38,8 & 36,8 & 37,0 \\
\hline $\begin{array}{l}\text { Enfermedad } \\
\text { cerebrovascular }\end{array}$ & 25,5 & 22,9 & 27,9 \\
\hline Cirrosis hepática & 23,2 & 27,6 & 25,8 \\
\hline Afecciones perinatales & 22,4 & 22,2 & 21,4 \\
\hline Neumonia e influenza & 20,8 & 21,3 & 20,7 \\
\hline Infecciones intestinales & 14,9 & 28,4 & 6,3 \\
\hline Deficiencias nutricionales & 12,3 & 17,1 & 5,7 \\
\hline
\end{tabular}

1 Tasa por 100 mil habitantes;

2 Incluye a: Chiapas, Guerrero, Hidalgo, Oaxaca, Puebla, Veracruz;

3 Incluye a: Baja California, Distrito Federal y Nuevo León

Fuente: Secretaría de Salud, 1994; OMS, 1998.

Tabla 5

Evolución de la mortalidad por causas seleccionadas. México, 1980-1997.

\begin{tabular}{|c|c|c|c|}
\hline Mortalidad & 1980 & 1990 & 1997 \\
\hline General1 & 6,2 & 5,1 & 4,6 \\
\hline Infantil2 & 40,3 & 23,9 & 16,4 \\
\hline Materna 3 & 9,0 & 5,4 & 4,7 \\
\hline Enfermedades prevenibles por vacunación 4 & 16,6 & 2,0 & 0,6 \\
\hline Enteritis 5 & 61,6 & 26,5 & 7,8 \\
\hline Neumonías 5 & 54,0 & 26,0 & 21,0 \\
\hline Neoplasias 5 & 40,5 & 49,1 & 54,1 \\
\hline Enfermedad isquémica del corazón5 & 23,3 & 28,9 & 44,9 \\
\hline Diabetes mellitus 5 & 21,9 & 30,8 & 38,0 \\
\hline Accidentes 5 & 69,4 & 71,6 & 37,9 \\
\hline
\end{tabular}

1 Defunciones por todas las causas por mil habitantes;

2 Defunciones en menores de 1 año por mil nacidos vivos registrados;

3 Defunciones por causas maternas por 10 mil nacidos vivos registrados:

4 Total de defunciones por sarampión, difteria, tosferina, tétanos

y poliomielitis por 100 mil;

5 Defunciones por causa específica por 100 mil habitantes

Fuente: COPLAMAR, 1982; Secretaría de Salud, 1984b, 1994; INEGI, 1998. 
das a los intereses electorales del Partido Revolucionario Institucional (PRI), además de dar una respuesta más propagandística que resolutiva, dado lo limitado de los recursos para financiar un conjunto muy heterogéneo de actividades (Consejo Consultivo del Pronasol, 1990). En este sexenio, se edita un populismo de nuevo tipo, prefigurando una política social de beneficencia que no interfiere con el desarrollo del proyecto de modernización económica y que, simultáneamente, mediatiza el descontento social exacerbado por el acelerado deterioro de las condiciones de vida de la mayor parte de la población. La acción del ejecutivo responde a las demandas populares, pero los beneficios son materializados como dádivas y no como derechos. Se legitima el discurso de "combate a la pobreza" y, en salud, se refuerza la atención masificada y barata dirigida a los grupos marginados rurales y urbanos, que no garantiza sino parte de los servicios de primer nivel de atención. En “compensación”, es en estos grupos donde se focalizan los programas de autocuidado y de educación para la salud (Kumate, 1989).

El énfasis sobre el autocuidado, la responsabilidad individual en la generación de la enfermedad (culpar a la víctima) y los programas para el fomento de la cultura en salud, orientados hacia los grupos más vulnerables que carecen de otras opciones de atención, caracterizan al Programa Nacional de Salud 1990-1994.

La política de salud en este sexenio justifica la focalización de los recursos y las acciones con un discurso sobre los beneficios de la aplicación selectiva del gasto social en momentos de crisis económica y de recursos financieros escasos. Según la visión oficial - a pesar de la crisis -, la selectividad permite mantener el acceso de los grupos más vulnerables a ciertos beneficios básicos, a través de la jerarquización de las zonas marginadas y de la correcta selección de los beneficiarios (Poder Ejecutivo Federal, 1990).

Así, en la década de los 80's y primera mitad de los 90's, en forma paralela a un discurso oficial que proclama la universalidad, la equidad y la solidaridad en la atención a la salud, los servicios y los programas transitan de propuestas integrales, incluyentes e intersectoriales a planteamientos de acción selectiva y focalizada a través de medidas epidemiológicas puntuales dirigidas a la población pobre.

El proceso de reorganización sanitaria y, más específicamente, la descentralización de los servicios de atención a población abierta juegan un papel muy importante en esta transformación, por lo que las instituciones y pro- gramas que atienden a la población sin seguridad social (fundamentalmente la Secretaría de Salud y el IMSS-Solidaridad) se constituyen en la punta de lanza de esta estrategia de neobeneficencia sanitaria.

\section{El periodo 1994-2000}

La reforma del campo sanitario iniciada en 1982 e impulsada durante el gobierno de Salinas de Gortari (1988-1994), en el sexenio encabezado por Ernesto Zedillo, vive una fase de continuidad y profundización a través de dos vertientes: la transformación de la seguridad social mediante las modificaciones a la Ley del Instituto Mexicano del Seguro Social (IMSS, 1997); y la reorganización de los servicios que atienden a la población no derechohabiente, contenidas en la propuesta de reforma del sector salud 1995-2000 (Programa de Reforma del Sector Salud, 1995).

En el primer caso, la nueva ley elimina el papel del IMSS como responsable único de la administración de fondos y pagador de las pensiones, al asignar estas tareas a las Administradoras de Fondos de Retiro (Afores) y a las aseguradoras, legaliza la administración y el control privado sobre los fondos de pensiones y sienta las bases para la transferencia masiva de fondos del sector público al privado, el fortalecimiento de los grupos financieros y la mercantilización del sistema de pensiones (Laurell, 1996; Ulloa, 1996).

En la misma lógica, la propuesta de reversión de cuotas en los servicios de atención a la salud - en donde el seguro público tendría que pagar a las empresas que optaran por la atención médica privada para sus trabajadores impulsa la privatización dentro del sistema de aseguramiento y sienta las bases para el éxodo de cotizantes de mayores ingresos hacia los servicios privados. En tanto estos cotizantes (alrededor de $20 \%$ ) aportan el $45 \%$ de los recursos cotizados por los trabajadores (Laurell, 1995), se preveen una profundización del desfinanciamiento de los servicios médicos del IMSS y una selección adversa para la institución pública, que se quedará con los trabajadores de menores ingresos y mayores riesgos, así como con aquellos excluidos de la atención privada por la complejidad, costo y/o cronicidad de su patología (Barreiro, 1996). En conjunto, se trata de una transformación que sustituye la concepción constitucional pública, solidaria e integral, de la seguridad social, por otra individualista con una fuerte tendencia privatizadora (De Buen, 1996). Como ejemplo del atractivo 
mercado que se abre para la inversión en salud, los seguros privados de gastos médicos mayores crecen de manera sostenida, representando para 1995 el 8\% del total de ventas de las compañías aseguradoras, con un total de 1,67 millones de asegurados. En igual forma, aumenta la proporción de los servicios médicos privados en el producto interno bruto de los servicios médicos (Laurell, 1997).

En el segundo caso y complementario a este proceso de fragmentación de la seguridad social y de mercantilización de la atención a la salud, se inscriben las propuestas para acelerar la reorganización de la atención médico-sanitaria de las instituciones públicas que atienden a población no asegurada. En estas instituciones, se plantea que los fondos públicos deben canalizarse a financiar únicamente servicios básicos de salud y permitir y alentar la conformación de mercados estables en donde la resolución de las necesidades de atención médica se realice en forma privada (Laurell \& López, 1996).

El Programa de Reforma del Sector Salud 1995-2000 propone la descentralización de los servicios de salud y la implementación de un paquete de servicios básicos como ejes centrales para la reorganización de las instituciones públicas que atienden a población sin seguridad social (Secretaría de Salud, 1995).

El proceso de descentralización propuesto como eje de la reforma del sector - al igual que su antecedente de los años ochenta - plantea la entrega de las unidades de la Secretaría de Salud (Ssa) y del Programa IMSS-Solidaridad a los gobiernos estatales, con el propósito de conformar Sistemas Estatales de Salud (SESA). Reedita las consideraciones sobre las necesidades y ventajas de descentralizar y expresan objetivos semejantes a los enarbolados en aquel periodo (1984-1988) (Zedillo, 1996). Sin embargo, está ausente un balance crítico sobre lo ocurrido con las 14 entidades federativas que se descentralizan entre 1985 y 1987, en donde en realidad lo que se concreta es una desconcentración operativa y - contra lo planteado en el discurso - se agudizan diversos problemas en el sector salud (López, 1996).

En los 90's, recuperando la visión de la atención primaria selectiva (Walsh \& Warren, 1979), las agencias financieras supranacionales (Banco Mundial y Banco Interamericano de Desarrollo, principalmente) plantean reorientar los recursos hacia las intervenciones que cuestan poco y que tienen alto impacto sobre algunas causas de mortalidad, de tal forma que los gobiernos financien un conjunto limitado de medidas de salud pública y de servicios clí- nicos esenciales dirigidos fundamentalmente a la población pobre, sin incrementos importantes del gasto público en salud (Banco Mundial, 1993).

La selectividad de la acción pública en salud no se limita a las acciones puntuales, sino que incluye también a las poblaciones. En el sexenio zedillista, los programas como el PASE (Programa de Alimentación, Salud y Educación), convertido posteriormente en PROGRESA (Programa de Educación, Salud y Alimentación), proponen restringir la actividad a los grupos pobres y, al interior de la población así etiquetada, focalizar - aún más - su quehacer en algunos subgrupos. Las propuestas de capacitación e información a las mujeres pobres, los programas para fomentar la escolaridad de las niñas pobres y el tratamiento de enfermedades comunes graves de la población infantil pobre se inscriben en esta lógica (Banco Mundial, 1993).

En México - en este periodo -, se determinan inicialmente 12 acciones constitutivas del paquete de servicios básicos, que se limitan a: saneamiento básico a nivel familiar; manejo de casos de diarrea en el hogar; tratamientos antiparasitarios a las familias; identificación de signos de alarma de infecciones respiratorias agudas y referencia a unidades de atención médica; prevención y control de tuberculosis pulmonar; prevención y control de hipertensión arterial y diabetes mellitus; inmunizaciones; vigilancia de la nutrición y crecimiento del niño; servicios de planificación familiar; atención prenatal, del parto y puerperio; prevención de accidentes y manejo inicial de lesiones, así como participación social (Secretaría de Salud, 1995). A principios de 1998, por la presión ejercida por grupos ciudadanos y redes de mujeres, se incorporan la prevención y el control del cáncer cervicouterino, como treceavo componente del paquete básico de salud.

La propuesta de paquete básico contrasta con la perspectiva integral planteada por la estrategia de Atención Primaria a la Salud (APS) (OMS, 1978), que en México sustenta la creación del Programa IMSS-Solidaridad, en 1979. En este programa, la salud se concibe como resultado de las condiciones vida y de trabajo de las poblaciones y se plantea su modificación, no sólo a través de la acción médica, sino también y esencialmente por medio del quehacer sanitario y de la transformación social (López, 1995). Esta concepción se concreta en un conjunto de servicios y opciones de atención ambulatoria y de hospitalización, para la población históricamente más excluida (campesinos e indígenas) (IMSS-Solidaridad, 1990b). 
En la medida que las propuestas de reorganización de las instituciones públicas de salud se inscriben en la lógica neoliberal de atención para pobres y se subordinan a las necesidades de la política macroeconómica, en los hechos tanto la descentralización como el paquete de servicios básicos restringen el papel del Estado como financiador y productor de servicios. Al orientar los fondos públicos para costear únicamente las intervenciones contenidas en el paquete básico, desde el gobierno se apoya el desmantelamiento del "monopolio" estatal de la salud y se "liberan" mercados antes vedados para la inversión privada con fines de lucro (Laurell \& López, 1996). En esta dinámica, tanto el discurso de la transición en salud, como la medición de las necesidades de salud y su priorización a través de ejercicios de costo-efectividad sirven de pretexto para apuntalar propuestas excluyentes en la atención, justifican las respuestas bipolares frente al fenómeno salud/enfermedad, mediante accesos diferenciales a las intervenciones y contribuyen a la difusión de las concepciones hegemónicas acerca de la salud, la enfermedad y la muerte, que insisten en que la enfermedad es resultado de conductas individuales y familiares insanas (estilos de vida poco saludables) y que su modificación es, ante todo, un problema de eficiencia técnica y de racionalidad administrativa (Blanco \& Rivera, 1994).

\section{Recorte y recomposición del financiamiento público del sector salud}

Mientras los principales objetivos de los Programas Nacionales de Salud 1984-1988, 19901994 (Poder Ejecutivo Federal, 1984, 1990) y del Programa de Reforma del Sector Salud 19952000 (Programa de Reforma del Sector Salud, 1995) son: ampliar la cobertura de los servicios de salud, dando prioridad a los núcleos rurales y urbanos más desprotegidos; homogeneizar y elevar la calidad en la prestación de los servicios de salud, atendiendo sobre todo problemas prioritarios y con especial énfasis en las acciones preventivas e impulsar la protección a todos los mexicanos, brindando servicios y prestaciones oportunos, eficaces, equitativos y humanitarios que coadyuven efectivamente al mejoramiento de sus condiciones de bienestar social, la orientación y el monto de los recursos financieros muestran otra cara de la modernidad sanitaria.

Así, en el periodo 1980-1988, la participación del gasto en salud y seguridad social como proporción del Producto Interno Bruto
(PIB) disminuye de 2,5\% a 1,3\% (Vásquez et al., 1988). En este periodo se produce la recomposición más drástica, pues el gasto en salud no sólo disminuye aceleradamente respecto al PIB, sino que su participación como proporción del financiamiento del sector público, también desciende de 6,2\% a 2,5\% (Vásquez et al., 1988). Así mismo, se reduce el presupuesto destinado a financiar los sistemas de atención a población sin seguridad social (de 21,6\% a $17,5 \%$ del gasto sectorial) y decrecen los recursos públicos para apoyar el segundo y el tercer nivel de atención (de 53,9\% a 44,9\% del gasto destinado a los servicios de atención curativa) (López \& Blanco, 1993). En el periodo 1989-94, se observa un incremento mínimo del gasto público en salud, sin recuperar los niveles previos a 1982, representando, para 1994, sólo el 1,7\% del PIB (Salinas-de-Gortari, 1994). La irrupción - en diciembre de 1994 - de una nueva crisis financiera vuelve a impactar negativamente el financiamiento del sector salud y, para 1995 y 1996, se tienen los niveles más bajos de la década.

En términos generales, de 1982 a 1998, se mantienen las tendencias en la reducción de los fondos destinados al mantenimiento de equipo e instalaciones, en la disminución de los recursos percápita para la atención a la salud de la población sin seguridad social y en la caída de los niveles salariales de los trabajadores de la salud. Además, es justamente en los periodos 1988-94 y 1994-2000 en donde la reestructuración del sector, especialmente la ampliación de cobertura con el paquete básico de salud y la reorganización de los servicios y beneficios de la seguridad social, está financiada (y condicionada) por los préstamos que el Banco Mundial y el Banco Interamericano de Desarrollo otorgan al gobierno mexicano.

La disminución y la pérdida de la centralidad de los fondos públicos destinados a la atención a la salud impactan negativamente a las instituciones del sector, reduciendo su capacidad resolutiva frente a los problemas de salud/enfermedad cada vez más diversos y complejos. Así mismo deslegitima su quehacer frente a la ciudadanía, refuerza las tesis neoliberales de que la ineficiencia, la mala calidad y el burocratismo son características inherentes a los servicios públicos y legitima las propuestas de mercantilización de lo público y de privatización de lo público rentable.

La política bipolar de neobeneficencia y mercantilización se hace más explícita en el periodo 1988-1994. Desde la definición del objetivo general del Programa Nacional de Salud 1990-1994 de “... impulsar la protección a todos 
los mexicanos ... con el concurso de las comunidades y los tres niveles de gobierno como medio eficaz para conseguir los recursos necesarios" (Poder Ejecutivo Federal, 1990:25), está presente la intencionalidad de desplazar el quehacer estatal constitucional hacia la población con el objeto de sumar esfuerzos financieros.

Sin embargo, la explicitación del retraimiento del Estado en el financiamiento de los servicios de salud y el progresivo traspaso de los costos de la atención médica a la población usuaria se hacen más evidentes en el programa de financiamiento. En este programa, se plantea una reestructuración financiera a través del incremento de los ingresos fiscales en los tres niveles de gobierno, la corresponsabilidad financiera de los gobiernos estatales y municipales y la diversificación de las fuentes de financiamiento (Poder Ejecutivo Federal, 1990). Esta reestructuración involucra financiamiento externo, convenios de cofinanciamiento, participación de instituciones privadas y aportaciones directas de la sociedad. Por ejemplo, "aumentar la contribución que en trabajo y en especie puede otorgar la comunidad... y establecer en la población comités o brigadas destinados a la remodelación, conservación y mantenimiento de los centros de salud" (Poder Ejecutivo Federal, 1990:29). De esta manera, bajo el amparo de un discurso de corresponsabilidad, se traslada parte del mantenimiento de los servicios y de los costos de la atención a la población usuaria.

El impacto diferencial de la recomposición del financiamiento sobre los distintos subsistemas del sector salud, en lugar de favorecer la homogenización en la calidad de los servicios, profundiza la desigual atención que recibe la población que accede a los distintos subsistemas. Aún cuando todas las instituciones públicas de salud son "castigadas" con el recorte presupuestal, el peso de la reducción se traslada con mayor intensidad a las instituciones que atienden a población sin seguridad social, a los programas de desarrollo de infraestructura y de prevención (p.e., los programas de control de paludismo, dengue, tuberculosis, lepra) en contradicción abierta con los objetivos planteados por la política declarativa (López \& Blanco, 1993).

Además, el proceso descentralizador de los años 80 fractura los mecanismos de redistribución del presupuesto federal, posibilitando que, paradójicamente, estados con mayores recursos absorban un porcentaje más elevado del apoyo financiero de la Federación. Por citar un ejemplo, los estados de México, Jalisco, Nuevo León y Tabasco, con poco más del $20 \%$ de la población nacional, concentraron en el trienio 1985-87 más del 50\% del total del financiamiento federal.

En los estados “pobres”, la falta de apoyo financiero provoca el deterioro acelerado de los servicios de salud y la necesidad de allegarse recursos de diversas fuentes, sentando las bases para la búsqueda de esquemas financieros diversificados, entre los que se destacan, impuestos estatales y municipales, pago por servicios, aumento de cuotas de recuperación y apertura a la inversión privada através de sistemas prepagados.

La descentralización de los 90's es aún mas limitada, pues en los hechos se reduce a desconcentrar a los niveles estatales y municipales la implementación de las acciones constitutivas del paquete básico de salud (López \& Blanco, 1997).

La recomposición del gasto social, la focalización de los recursos públicos en regiones y poblaciones "pobres”, la intervención selectiva a través de acciones de administración, más que de combate a la pobreza extrema, y la reorganización institucional con el fin de conservar un mínimo de servicios baratos y simplificados para los grupos más depauperados delinean una política social de neo-beneficencia.

En el otro polo, se conforma un sector privado muy activo, que crece aceleradamente durante los últimos 10 años, a través de la expansión de las aseguradoras y de las grandes corporaciones médicas.

Estas tendencias conforman dos mundos extremos, en donde los "pobres" tienen acceso a un financiamiento público mínimo que sólo garantiza un reducido número de acciones en salud. Recursos que son focalizados en regiones y poblaciones calificadas o credencializadas como pobres extremas, priorizadas por índice de marginalidad y con techos financieros predeterminados y un mundo de "clientes" que accede a una atención médica de calidad diferenciada según su capacidad de pago, a través de diversos mecanismos que garanticen la conformación de mercados estables (p.e., aseguramiento privado colectivo) y a un sector público flexibilizado que favorece la subrogación y la introducción de la lógica mercantil, a través de las formas de contrato público y del desarrollo de mercados internos (p.e., la propuesta de zonas médicas del IMSS y el impulso a las Instituciones de Seguros Especializadas en Salud - ISES -, semejantes a las Health Management Organization (HMO's) desarrolladas en los Estados Unidos. 


\section{Consideraciones finales}

En la modificación transexual de la política social, ésta se subordina a las necesidades del ajuste macroeconómico y - en la misma lógica - la política de salud restringe la acción pública a modelos asistenciales y selectivos que se concretan a través de paquetes de servicios básicos, justificados desde una perspectiva tecnocrática en términos de costo-efectividad, configurando así el polo de neo-beneficencia.

En forma paralela y como estrategia central para ordenar al sector salud en su conjunto, se desarrolla una activa promoción gubernamental orientada a conformar mercados estables para la atención a la salud. Es decir, la intervención estatal se orienta hacia los pobres en una lógica de beneficencia, mientras que, para la población "no pobre", el acceso a diversos servicios y la resolución de sus necesidades se plantean a través del mercado.

Estas transformaciones de las políticas social y de salud se articulan sin contradicción con la perspectiva gubernamental sobre las "virtudes" del mercado en la vida social y económica, como el mejor asignador de recursos e incentivador de la competencia, de la diversidad y de la libre elección de los consumidores; con la visión del quehacer público limitado a las acciones compensatorias y focalizadas en la población pobre, así como en el interés del sector privado de "romper" los monopolios públicos e invertir en el sector salud, por su creciente importancia económica y financiera.

En este proceso, los actores tradicionales del campo sanitario son desplazados (funcionarios y trabajadores de la salud), emergen nuevos actores como las agencias financieras multinacionales y las compañías aseguradoras, con un papel crecientemente protagónico, y otros, como la gran empresa médica, se fortalecen.

En términos de la recomposición financiera del sector salud, se produce una pérdida de la centralidad del financiamiento público, pues se pretende que estos recursos se canalicen exclusivamente a financiar intervenciones costoefectivas dirigidas sobre todo a la población pobre. Adicionalmente, el gobierno reduce el gasto social como parte de su estrategia general para disminuir el déficit fiscal, mantener unas finanzas sanas y liberar recursos para sus prioridades reales, que, en la década de los ochenta, son el pago puntual de los intereses de la deuda externa y, para los noventa, se suman a esta prioridad los rescates - con fondos públicos - de los inversionistas privados que fracasaron en el negocio de las autopistas y del sistema bancario.
Así, al desfinanciamiento prolongado al que han sido sometidas las instituciones públicas de salud y a la ausencia de "rescates" que limiten su deterioro se suman propuestas de reorganización financiera que, lejos de fortalecerlas, las debilitan y se privilegian formas regresivas de asignación presupuestal.

Los recursos públicos limitados sólo a financiar paquetes básicos de salud, el traslado de los costos de la atención a los usuarios y la privatización de lo público rentable muestran un retraimiento selectivo del Estado en la cuestión sanitaria y el viraje neoliberal de la política de salud. Este modelo de política de salud es regresivo y excluye a grandes grupos de población de servicios de salud de calidad. En esta dinámica, la pérdida de centralidad del presupuesto público y la focalización de los recursos a través de programas como el PASE o, más recientemente, el PROGRESA, financiados con préstamos de organismos supranacionales, lejos de reforzar la estructuración de un sistema de salud de calidad, equitativo y universalista, contribuyen a acelerar los procesos de fragmentación y mercantilización de las instituciones públicas de salud y a profundizar el sistema dual y polarizado que se ha conformado en los últimos 17 años. 


\section{Referencias}

BANCO MUNDIAL, 1993. Informe sobre el Desarrollo Mundial 1993. Invertir en Salud. Washington, D.C.: Banco Mundial.

BARBA, C., 1997. Distribución del ingreso, crecimiento económico y democracia en México: Alternativas de política social. In: Libro 5. La Política Social. El Debate Nacional (E. Valencia \& C. Barba, eds.), pp. 61-97, México, D.F.: Editorial Diana.

BARREIRO, R., 1996. Evaluación de los riesgos de la reforma. In: Alternativas de Reforma de la Seguridad Social (L. Mussot, ed.), pp. 77-89, México, D. F.: Fundación Ebert/Universidad Autónoma Metropolitana-Xochimilco.

BLANCO, J. \& LOPEZ, O., 1990. Política de salud e impacto epidemiológico. Salud Problema, 20: 19-28.

BLANCO, J. \& RIVERA, J. A., 1994. La carga global de morbilidad. Justificación científico-técnica de invertir en salud. In: Tendencias y Alternativas en el Sector Salud (C. Laurell, ed.), pp. 111-128, México, D.F.: Fundación Ebert.

BLANCO, J.; RIVERA, J. A.; LOPEZ, O. \& RUEDA, F., 1998. Calidad de vida y salud en la Zona Metropolitana de la Ciudad de México. In: Ciudades Latinoamericanas: Modernización y Pobreza. (A. Ziccardi, \& S. Reyes, eds.), pp. 187-200, México, D.F.: Programa Universitario de Estudios sobre la Ciudad/Instituto de Investigaciones Sociales, Universidad Nacional Autónoma de México.

BOLTVINIK, J., 2000a. Debate, desigualdad y pobreza. La Jornada, 28 abr., p. 27.

BOLTVINIK, J., 2000b. Nada que festejar. La Jornada, 5 may., p. 30.

CONSEJO CONSULTIVO DEL PRONASOL, 1990. El Combate a la Pobreza: Lineamientos Programáticos. México, D.F.: El Nacional.

COPLAMAR (Coordinación General del Plan Nacional de Zonas Deprimidas y Grupos Marginados), 1982. Necesidades Esenciales en México, No. 4, Salud. México, D.F: COPLAMAR.

DE BUEN, N., 1996. Principios constitucionales de la seguridad social mexicana en el contexto de la globalización. In: Alternativas de Reforma de la Seguridad Social (L. Mussot, ed.), pp. 17-39, México, D.F.: Fundación Ebert/Universidad Autónoma Metropolitana-Xochimilco.

ESPINOZA, J., 1986. Descentralización y Desconcentración de las Funciones de Regulación, Control y Fomento Sanitario. Memoria del Seminario-Taller de Capacitación para la Regulación, Control y Fomento Sanitarios. México, D.F.: Secretaría de Salud.

FERNANDEZ-DE-CASTRO, J., 1988. Panorama Histórico y Epidemiológico del Paludismo en México. México, D.F: Secretaría de Salud.

GONZALEZ, M. \& LEYVA, R., 1988. Economía Política de la Descentralización de la Salud en México: Un Análisis Comparativo de sus Consecuencias en la Planeación Sectorial y Regional. México, D.F.: Centro de Investigaciones en Salud Pública, Instituto Nacional de Salud Pública.

GRUPO FINANCIERO BANAMEX-ACCIVAL, 2000. Informe. México, D.F.: Grupo Financiero BanamexAccival.
IMSS (Instituto Mexicano del Seguro Social), 1997. Ley del Instituto Mexicano del Seguro Social. México, D.F.: IMSS.

IMSS-Solidaridad (Instituto Mexicano del Seguro Social-Solidaridad), 1990a. Boletín Informativo 8. México, D.F.: IMSS-Solidaridad.

IMSS-Solidaridad (Instituto Mexicano del Seguro Social-Solidaridad), 1990b. Convenio para el Establecimiento de Servicios de Salud en el Medio Rural. México, D.F.: Palacio Nacional.

INEGI (Instituto Nacional de Estadística, Geografía y Informática), 1998. Estadísticas Vitales. México, D.F.: Secretaría de Salud.

KUMATE, J., 1989. Programa Nacional de Promoción y Cuidado de la Salud. In: Reunión del Gabinete de Bienestar Social, Memorias, pp. 1-5, México, D.F:Secretaría de Salud.

LAURELL, C., 1992. La política social en el proyecto neoliberal. Necesidades económicas y realidades sociopolíticas. Cuadernos Médico-Sociales, 60:1112.

LAURELL, C., 1995. Para pensar una política social alternativa. In: Estado y Políticas Sociales Después del Ajuste. Debates y Alternativas (C. Vilas, ed.), pp. 183-187, México, D.F.: Universidad Autónoma Metropolitana-Xochimilco/Editorial Nueva Sociedad.

LAURELL, C., 1996. No Hay Pierde: Todos Pierden. La Reforma a la Ley del IMSS. México, D.F.: Instituto de la Revolución Democrática.

LAURELL, C., 1997. La Reforma Contra la Salud y la Seguridad Social. México, D.F.: Editorial ERA/ Fundación Ebert.

LAURELL, C. \& LOPEZ, O., 1996. Market commodities and poverty relief. The World Bank proposal for health. International Journal of Health Services, 26:1-18.

LOPEZ, O., 1990. La Política de Salud en México 198288. Una Visión Crítica. Tesis de Maestría, México, D.F.: Maestría en Medicina Social, Universidad Autónoma Metropolitana-Xochimilco.

LOPEZ, O.,1992. La política de salud en México. ¿Un ejemplo de liberalismo social? In: Estado y Politicas Sociales en el Neoliberalismo (C. Laurell, ed.), pp. 165-183, México, D.F.: Fundación Ebert.

LOPEZ, O., 1995. De la solidaridad social a la neobeneficencia sanitaria, un riesgoso camino para el IMSS-Solidaridad. In: Memorias del Foro de Beneficios, Costos y Financiamiento de la Seguridad Social, pp. 88-89, México, D.F.: Cámara de Diputados.

LOPEZ, O.,1996. La estrategia descentralizadora en una política social incluyente. In: Hacia una Política Social Alternativa (C. Laurell, ed.), pp. 129140, México, D.F.: Fundación Ebert/Instituto de Estudios de la Revolución Democrática.

LOPEZ, O. \& BLANCO, J., 1993. La Modernización Neoliberal en Salud. México en los Ochenta. México, D.F.: Universidad Autónoma MetropolitanaXochimilco.

LOPEZ, O. \& BLANCO, J., 1997. Los retos en salud para el fin de siglo. In: Libro 5. La Política Social. El Debate Nacional (E. Valencia \& C. Barba, eds.), pp. 183-206, México, D.F.: Editorial Diana. 
OMS (Organización Mundial de la Salud), 1978. Declaración de Alma Ata. Reporte de la Conferencia Internacional sobre Atención Primaria a la Salud. Génova: OMS.

OMS (Organización Mundial de la Salud), 1998. La Salud de las Américas 1998. Perfil de Salud del País: México. Ginebra: OMS.

PODER EJECUTIVO FEDERAL, 1988. Plan Nacional de Desarrollo 1984-1988. México, D.F.: Poder Ejecutivo Federal.

PODER EJECUTIVO FEDERAL, 1994. Programa Nacional de Salud 1990-1994. México, D.F.: Poder Ejecutivo Federal.

PROGRAMA DE REFORMA DEL SECTOR SALUD, 1995. Programa de Reforma del Sector Salud 1995-2000. México, D.F.: Poder Ejecutivo Federal.

SALINAS-DE-GORTARI, C., 1991.Tercer Informe de Gobierno. México, D.F.: Poder Ejecutivo Federal.

SALINAS-DE-GORTARI, C., 1994. Sexto Informe de Gobierno, Anexo Estadístico. México, D.F.: Poder Ejecutivo Federal.

SECRETARIA DE SALUD, 1984a. Ley General de Salud. México, D.F.: Secretaría de Salud.

SECRETARIA DE SALUD, 1984b. Programa Sectorial de Mediano Plazo. Programa Nacional de Salud, 1984-1998. México, D.F.: Secretaría de Salud.
SECRETARIA DE SALUD, 1994. Contexto Actual. Mortalidad 1993. Aspectos Relevantes. Perfiles Estadisticos. Series Monográficas 8, México, D.F.: Secretaría de Salud.

SOBERON, A. \& KUMATE, J., 1988. La Salud en México: Testimonios 1988. El Cambio Estructural. México, D.F.: Secretaría de Salud/Fondo de Cultura Económica.

SORIA, V. \& FARFAN, G., 1990. El deterioro del bienestar social en México. In: Testimonios de la Crisis. 4. Los Saldos del Sexenio 1982-1988 (E. Gutiérrez, ed.), pp. 139-177. México, D.F.: Editorial Siglo XXI.

ULLOA, O., 1996. Nueva Ley del Seguro Social: La reforma previsional de fin de siglo. El Cotidiano, 76:21-26.

VASQUEZ, S.; HURTADO, I.; GUZMAN, S. \& NOBARA R., 1988. Notas sobre la estructura y evolución del presupuesto programático ejercido por las instituciones que conforman el sector salud. 19801987. Salud Problema, 15:37-46.

WALSH, J. \& WARREN, K., 1979. Selective primary health care: An interim strategy for disease control in developing countries. New England Journal of Medicine, 301:967-974.

ZEDILLO, E., 1996. Palabras del presidente en el acto de firma de los convenios de descentralización. La Jornada, 21 ago., p. 10. 